\title{
miR-375 is upregulated in acquired paclitaxel resistance in cervical cancer
}

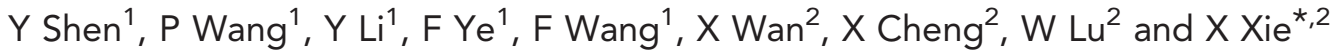 \\ ${ }^{1}$ Women's Reproductive Health Laboratory of Zhejiang Province, Women's Hospital, School of Medicine, Zhejiang University, \\ Hangzhou, China and 'Department of Gynecologic Oncology, Women's Hospital, School of Medicine, Zhejiang University, \\ Hangzhou 310006, China
}

Background: Chemo-resistance is one of the key causal factors in cancer death and emerging evidences suggest that microRNAs (miRNAs) have critical roles in the regulation of chemo-sensitivity in cancers. Cervical cancer is one of the most common malignancies in women and insensitive to chemotherapy clinically.

Methods: The differentially expressed miRNAs in cervical squamous cell carcinoma tissues were screened by using a microarray platform ( $\mu$ Paraflo Sanger miRBase release 13.0). The expression of miR-375 was determined by stem-loop RT-PCR using 23 clinical cervical cancer samples and 2 cervical cancer cell lines. We exogenously upregulated miR-375 expression in SiHa and Caski cells using a pre-miRNA lentiviral vector transfection and observed its impact on paclitaxel sensitivity using MTS. The cells that stably overexpressed miR-375 were subcutaneously injected into mice to determine tumour growth and chemo-sensitivity in vivo.

Results: Twenty-one differentially expressed miRNAs were found by miRNA microarray between pro- and post-paclitaxel cervical cancer tissues. Of those, miR-375 showed consistent high expression levels across paclitaxel-treated cervical cells and tissues. Paclitaxel induced upregulated miR-375 expression in a clear dose-dependent manner. Forced overexpression of miR-375 in cervical cancer cells decreased paclitaxel sensitivity in vitro and in vivo.

Conclusion: Collectively, our results suggest that miR-375 might be a therapeutic target in paclitaxel-resistant cervical cancer.

Chemotherapy is an extremely important therapeutic strategy in cancers, but chemo-resistance remains one of the key causal factors in cancer death and has confused worldwide scientists and oncologists longtime (Wang et al, 2010). As we know, the majority of tumours responds well initially to chemotherapy, but eventually develops resistance following treatment (Wendt et al, 2010). Chemotherapy agents can facilitate the phenotypic transformation in residual surviving cancer cells into a highly mobile and resistive phenotype (Dean et al, 2005; Ranganathan et al, 2006). Insight into the molecular mechanism by which tumours overcome the toxicity of drugs is a critical step in preventing or reversing chemo-resistance.

MicroRNAs (miRNAs) are small 20-23 nucleotide non-coding RNAs that act as important gene regulators in human genomes and their aberrant expression may promote not only tumorigenesis but also tumour aggressiveness (Chang et al, 2011). Recently, the association between miRNA expression and chemo-sensitivity in cancer is closely noticed, which is regarded for predicting chemosensitivity in cancers. Indeed, some miRNAs have been found to predict sensitivity to anticancer treatment, such as Let-7g and miR$181 \mathrm{~b}$ that are strongly associated with response to 5-fluorouracilbased antimetabolite S-1 (Nakajima et al, 2006). Functional studies show that such miRNAs can modulate chemo-sensitivity, for instance, miR-21 inhibition increases sensitivity to gemcitabine in cholangiocarcinoma cell (Meng et al, 2006), miR-34 restoration in p53-deficient human gastric cancer cells induces increased chemosensitivity (Kim et al, 2011). In addition, chemotherapy also inversely alters miRNA expression and mediates the drug sensitivity in cancer cells in turn. For example, cis-platinum significantly reduces miR-30a expression in cancer cells which in turn strongly inhibits cis-platinum-induced apoptosis (Zou et al, 2012). Thus, there is a complicated regulating feedback loop between miRNA and chemo-resistance in cancers. Anyway, it could be believed that a break of such a loop would not only

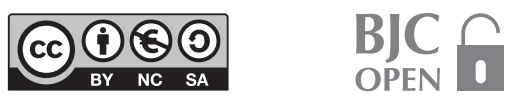


overcome cancer chemo-resistance but also provide important new therapeutic targets. However, miRNAs regulating drug sensitivity are varied in different kinds of cancer and different anticancer agents. Therefore, the identification of the role of a specific miRNA modulating a specific anticancer drug in a specific cancer has a clinical significance.

Cervical cancer is the third most common gynaecologic cancer in women worldwide (Wilson et al, 2004). Chemotherapy is merely a complemented treatment to surgery or radiation therapy for the cancer due to its insensitivity to anticancer drugs (Kim et al, 2011). Although cervical cancer screening has been globally popularised, there still are large numbers of advanced diseases, especially in developing countries including China. Paclitaxel combined with platinum chemotherapy is a recommended therapeutic regimen for advanced cervical cancer by authorised professional organisations in the world, but the 5-year survival rate is only $30-60 \%$ (Yavas and Yavas, 2012). Thus, it is significant to identify a novel therapeutic target that has a key role in regulating cervical cancer cells to chemosensitivity for improving the prognosis of cervical cancer patients.

In this study, we first used high-throughput miRNA expression analysis to reveal miRNAs associated with chemo-sensitivity using cervical squamous cell carcinoma tissues treated with chemotherapeutic agent paclitaxel. Among 21 differentially expressed miRNAs, miR-375 showed consistent higher expression levels across paclitaxel-treated cervical cells and tissues, suggesting that miR-375 may be contribute to paclitaxel chemo-resistance. Then, the role of miR-375 in paclitaxel chemo-sensitivity was explored in cervical cancer tissues, cells, and mouse models.

\section{MATERIALS AND METHODS}

Patients and samples. The samples of cancer tissue were collected from 25 cervical squamous cell carcinoma patients with Federation of Gynecology and Obstetrics (2009) stage $\mathrm{IB}_{2}$ or IIA 2 (tumour size $>4 \mathrm{~cm}$ ) who initially underwent neo-adjuvant chemotherapy followed by type III radical hysterectomy between 1 November 2008 and 30 May 2010 in Women's Hospital, School of Medicine, Zhejiang University, China. No patient received radiotherapy before the tissues were obtained. The collection of all samples was approved by the Ethical Committee for Clinical Research of the Hospital and informed consents were obtained.

All patients underwent two cycles of intravenous neo-adjuvant chemotherapy (paclitaxel $135 \mathrm{mg} \mathrm{m}^{-2}$ and cisplatin $75 \mathrm{mg} \mathrm{m}^{-2}$, 3 -week interval) before surgery. The effect of chemotherapy was evaluated for tumour size measured at the initial diagnostic procedure and just after chemotherapy but before surgery under colposcopy according to the Response Evaluation Criteria in Solid Tumors (RECIST1.1) (Eisenhauer et al, 2009). Each patient will be assigned one of the following categories: complete response (CR), partial response $(\mathrm{PR})$, stable disease $(\mathrm{SD})$, and progression disease (PD). Then, we defined CR\&PR as chemo-sensitive and SD\&PD as chemo-resistance. Of all 25 patients, mean age was 36.8 years (range 20-65 years), 21 were chemo-sensitive and 4 were chemoresistance, 23 had pre- and post-chemotherapy samples and 2 only had pre-chemotherapy samples because the tumours achieved pathological remission. All the cervical cancer samples are Hybrid Capture II HPV Test (HCII) (Digene Corporation, Gaithersburg, MD, USA) positive. To obtain homogeneous and histological wellcharacterised samples for RNA analyses, the nature of the tissue and its specified composition were determined by an experienced pathologist. All the samples were immediately snap frozen and stored in liquid nitrogen $\left(-180^{\circ} \mathrm{C}\right)$ until RNA extraction.

Cell culture and chemotherapy inducement. Two human cervical cancer cell lines, $\mathrm{SiHa}$ and Caski, were purchased from the American Type Culture Collection (ATCC University Boulevard, Manassas, VA, USA) and preserved in our laboratory. Cell lines were cultured in RPMI-1640 medium supplemented with $10 \%(\mathrm{v} / \mathrm{v})$ fetal bovine serum in a humidified $\mathrm{CO}_{2}$ incubator at $37^{\circ} \mathrm{C}$. Paclitaxel was obtained from Bristol-Myers Squibb Ltd., respectively, used at a final concentration of 5 and $10 \mathrm{~nm}$. The cancer cell lines (SiHa and Caski) were seeded $3 \times 10^{5}$ per well in six-well plates and incubated overnight, and then treated with paclitaxel for $72 \mathrm{~h}$. After $72 \mathrm{~h}$ of induction, cells were harvested for further experimentation.

miRNA microarray assay. From the liquid nitrogen frozen tissues, we selected five paclitaxel-sensitive and four paclitaxelresistant cervical squamous cell carcinoma tissues collected before chemotherapy and four couples of self-paired (pre- and postchemotherapy samples) cervical squamous cell carcinoma tissues, sent to the United States LC science companies for miRNA isolation, quality control, chip hybridisation, and microarray data analysis after succeeding in extracting the total RNA. In LC Sciences, miRNA microarrays were performed on $\mu$ Paraflo Microfluidic Biochip (version 12.0; LC Sciences, Houston, TX, USA), each of which can detect 8273 miRNAs with multiple control probes for assessing qualities (Wang and Cheng, 2008). The fluorescence images of the chips were collected using a laser scanner (GenePix 4000B, Molecular Device, Sunnyvale, CA, USA) and digitised using Array-Pro image analysis software (Media Cybernetics, Bethesda, MD, USA). Data were analysed by first subtracting the background and then normalising the signals using a LOWESS filter (Locally weighted Regression) (Bolstad et al, 2003). ANOVA is first applied to produce an miRNA expression profile overview across all samples; then $T$-tests are performed to identify significantly differentiated miRNAs among all interested combinations of two groups.

RNA extraction and real-time RT-PCR. Total RNAs containing miRNAs were extracted from liquid nitrogen preserved tissues or the harvested cells using $1 \mathrm{ml}$ TRIZOL reagent (Invitrogen,

Table 1. The real-time PCR primers for miRNAs, RNU6, and EEF1A1

\begin{tabular}{|c|c|c|}
\hline Gene & Forward primer & Reverse primer \\
\hline hsa-miR-375 & 5'-AGCCGTTTGTTCGTTCGGCT-3' & 5'-GTGCAGGGTCCGAGGT-3' \\
\hline hsa-miR-424 & 5'-AGCGGCAGCAGCAATTCATC-3' & 5'-GTGCAGGGTCCGAGGT-3' \\
\hline hsa-miR-27a & 5'-CGCGCTTCACAGTGGCTAAG-3' & 5'-GTGCAGGGTCCGAGGT-3' \\
\hline hsa-miR-181b & 5'-CGGCGAACATTCATTGCTGT-3' & 5'-GTGCAGGGTCCGAGGT-3' \\
\hline hsa-miR-224 & 5'-AGCGGTGGCTCAGTTCAGCA-3' & 5'-GTGCAGGGTCCGAGGT-3' \\
\hline U6snRNA & 5'-CTCGCTTCGGCAGCACA-3' & 5'-AACGCTTCACGAATTTGCGT-3' \\
\hline EEF1A1 & 5'-TGCGGTGGGTGTCATCAAA-3' & 5'-AAGAGTGGGGTGGCAGGTATTG-3' \\
\hline
\end{tabular}


Carlsbad, CA, USA) following manufacturer's instructions. cDNA was synthesised with the PrimeScript RT reagent Kit (TaKaRa Otsu, Shiga, Japan). Quantitative RT-PCR (qRT-PCR) for miRNA and mRNA was performed as described previously (Wang et al, 2011), SYBR green real-time PCR was performed with an Applied Biosystems 7900HT Fast Real-time PCR system (ABI, Foster City, CA, USA) using the SYBR Premix Ex Taq (perfect real time) (TaKaRa Biotechnology, Otsu, Shiga, Japan). The U6 snRNA and EEF1A1 were used as endogenous control for miRNA and mRNA, respectively. The $\Delta \mathrm{Ct}$ method was used to determine relative quantization of miRNA and mRNA expression in samples, and the fold change was determined as $2^{-\Delta \Delta \mathrm{Ct}}$. The sequences of all primers are given in Tables 1 and 2 .

MTS assay. An in vitro paclitaxel chemo-sensitivity of cervical cancer cells ( $\mathrm{SiHa}$ and Caski) was evaluated using MTS assay. Cells were triplicately seeded in flat-bottomed 96-well plates at a density of $6 \times 10^{3}$ per well, cultured overnight, and supplemented with various paclitaxel doses $(0,5,10,20,50$, and $100 \mathrm{~nm})$. After 3 days, replaced the medium with $100 \mu \mathrm{l}$ fresh medium, $20 \mu \mathrm{l}$ CellTiter 96 Aqueous One Solution Reagent (Promega, Madison, WI, USA) was added to each well according to manufacturer's instructions. After $4 \mathrm{~h}$ in culture, the cell viability was determined by measuring the absorbance at $490 \mathrm{~nm}$ using a 550 BioRad plate-reader (Bio-Rad, Hertfordshire, UK).

Lentivirus construction and transfection. To generate miR-375 and Ecadherin overexpression stable transfectants, cervical cancer cells were transfected with lentiviral expressing vectors, and stable clones were selected with EGFP by flow cytometry. The plasmid construction and lentivirus package were completed in GENECHEM Company (Shanghai, China). The pre-miR-375 sequences were amplified and cloned into the pGCsil-GFP vector using the following primers: (Forward) hsa-pre-miR-375-XhoI-F, 5'ACCG CTCGAGCCCCGCGACGAGCCCCTCGC3' (Reverse) hsa-premi-R-375-MfeI-R, 5'ACCGCAATTGAAAAAAGCCTCACGC GAGCCGAACG3'. The has-CDH1(CDS) gene was amplified and cloned into a pGC-FU-GFP vector using the following primers: (Forward) $5^{\prime}$-AATTCCGTGGTGTTGTCG-3'; (Reverse) 5'-AAGGTCCGCTGGATTGAG-3'. Viruses packaging was performed in HEK 293T cells after the co-transfection of $20 \mathrm{mg}$ pGCsil-GFP-pre-miR-375 vector or pGC-GFP-CDH1 vector with $15 \mathrm{mg}$ of the packaging plasmid pHelper 1.0 Vector and $10 \mathrm{mg}$ of the envelope plasmid pHelper 2.0 vector using Lipofectamine 2000 (Life Technologies, Carlsbad, CA, USA). Viruses were harvested $48 \mathrm{~h}$ after transfection, and viral titers were determined. The titers of the viral used in this study were in the range of $5 \times 10^{8}$ $2 \times 10^{9} \mathrm{TU} \mathrm{ml}^{-1}$.

For stably transfected cells, cells were transfected with lentivirus at $30-50 \%$ confluency in six-well plates. Lentivirus vector with $2 \mathrm{mg} \mathrm{ml}^{-1}$ polybrene was added to the Opti-MEM medium, and $24 \mathrm{~h}$ later the vector containing media was replaced and changed to fresh culture media. An empty pGC FU-GFP-NC-LV vector was used as a negative control. An MOI of 5 was used for $\mathrm{SiHa}$ and an
MOI of 10 was used for Caski. After 4-7 days of transfection, stable clones were selected with GFP by flow cytometry and harvested for further experimentation.

Animal experiments. Female BALB/C nude mice of 2-4 weeks old were purchased from Shanghai Laboratory Animal Center (SLAC, Shanghai, China) and housed within a dedicated SPF facility at Laboratory Animal Center of Zhejiang University. The $\mathrm{SiHa}$ and Caski cells that stably overexpressed miR-375, negative, or blank controls were harvested, resuspended in serum-free medium after washing with PBS, and injected subcutaneously into the right-side axilla of each mouse (Caski $\left(6 \times 10^{6}\right)$ and $\mathrm{SiHa}$ $\left.\left(4 \times 10^{6}\right)\right)$, respectively. Once the mice developed palpable tumours, they were selected into the following treatment groups according to their closely matched tumour volumes: (1) overexpressing miR-375 received paclitaxel $(n=6)$; (2) negative control received paclitaxel $(n=7)$; (3) overexpressing miR-375 received saline $(n=5)$; and (4) negative control received saline $(n=7)$. The paclitaxel $\left(15 \mathrm{mg} \mathrm{kg}^{-1}\right)$ and the same quantity of normal saline were intraperitoneally injected once a week for 4 weeks. The health of the mice and evidence of tumour growth were examined every 3 days. Tumours were measured twice a week with a digital caliper. Tumour volume $\left(\mathrm{mm}^{3}\right)$ was calculated as Width $2 /$ Length. All mice were killed on day 7 after complete drug treatments. Subcutaneous tumours were surgically excised, weighed, photographed, sectioned, and stained with haematoxylin-eosin. The expressions of miR-375 in tumours were measured by real-time RT-PCR. All the experiments have been carried out with ethical committee approval and meet the standards required by the UKCCCR guidelines (Workman et al, 2010).

Statistical analysis. The experiments were repeated at least three times. Results are expressed as mean \pm s.e.m. An independent Student's $t$-test or an ANOVA was used to compare continuous variables. Pearson's correlation coefficient test was used to assess the correlation between two continuous variables. $P<0.05$ was considered as statistically significant.

\section{RESULT}

Screening and identification of miRNAs associated with paclitaxel resistance in cervical cancer. Considering the existence of intrinsic and acquired drug resistance, we simultaneously detected differentially expressed miRNAs in cervical squamous cell carcinoma tissues between paclitaxel sensitive and resistant and between pre- and post-chemotherapy by using a microarray platform that covered a total of 875 human miRNAs ( $\mu$ Paraflo Sanger miRBase release 13.0; www.mirbase.org) Each sample was confirmed to contain $>80 \%$ tumour tissues by histology. Totally, 21 differentially expressed miRNAs were found between self-paired pre- and postchemotherapy cancer tissues by ANOVA, including 13 downregulated and 8 upregulated miRNAs (Figure $1 \mathrm{~A}$ and $\mathrm{B}$ ), but no

\begin{tabular}{|c|c|}
\hline miRNA & Reverse Transcription primer \\
\hline hsa-miR-375 & 5'-GTCGTATCCAGTGCAGGGTCCGAGGTATTCGCACTGGATACGACTCACGC-3' \\
\hline hsa-miR-424 & 5'-GTCGTATCCAGTGCAGGGTCCGAGGTATTCGCACTGGATACGACTTCAAA-3' \\
\hline hsa-(xie)miR-27a & 5'-GTCGTATCCAGTGCAGGGTCCGAGGTATTCGCACTGGATACGACGCGGAA-3' \\
\hline hsa-miR-181b & 5'-GTCGTATCCAGTGCAGGGTCCGAGGTATTCGCACTGGATACGACACCCAC-3' \\
\hline hsa-miR-224 & 5'-GTCGTATCCAGTGCAGGGTCCGAGGTATTCGCACTGGATACGACCTGTTC-3' \\
\hline U6snRNA & 5'-AACGCTTCACGAATTTGCGT-3' \\
\hline
\end{tabular}


A
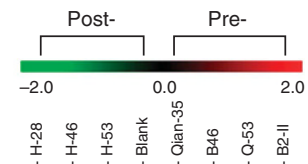

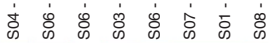
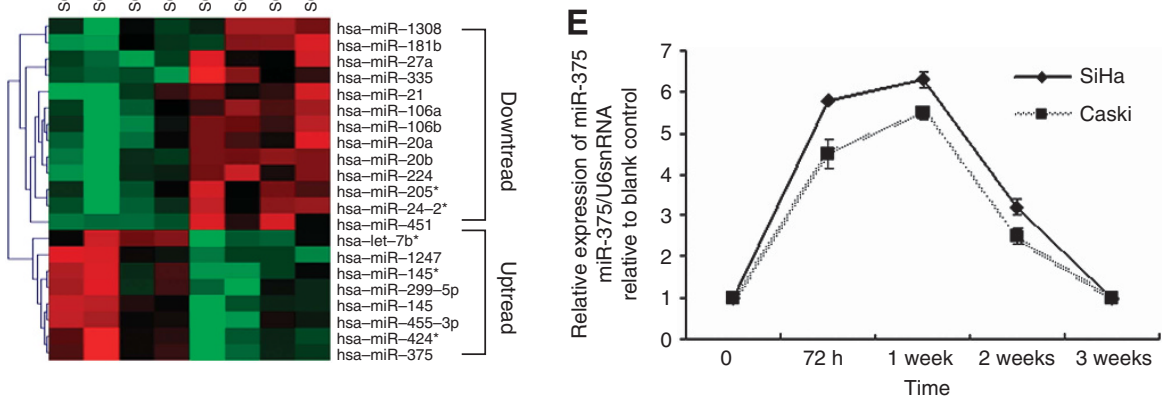

B
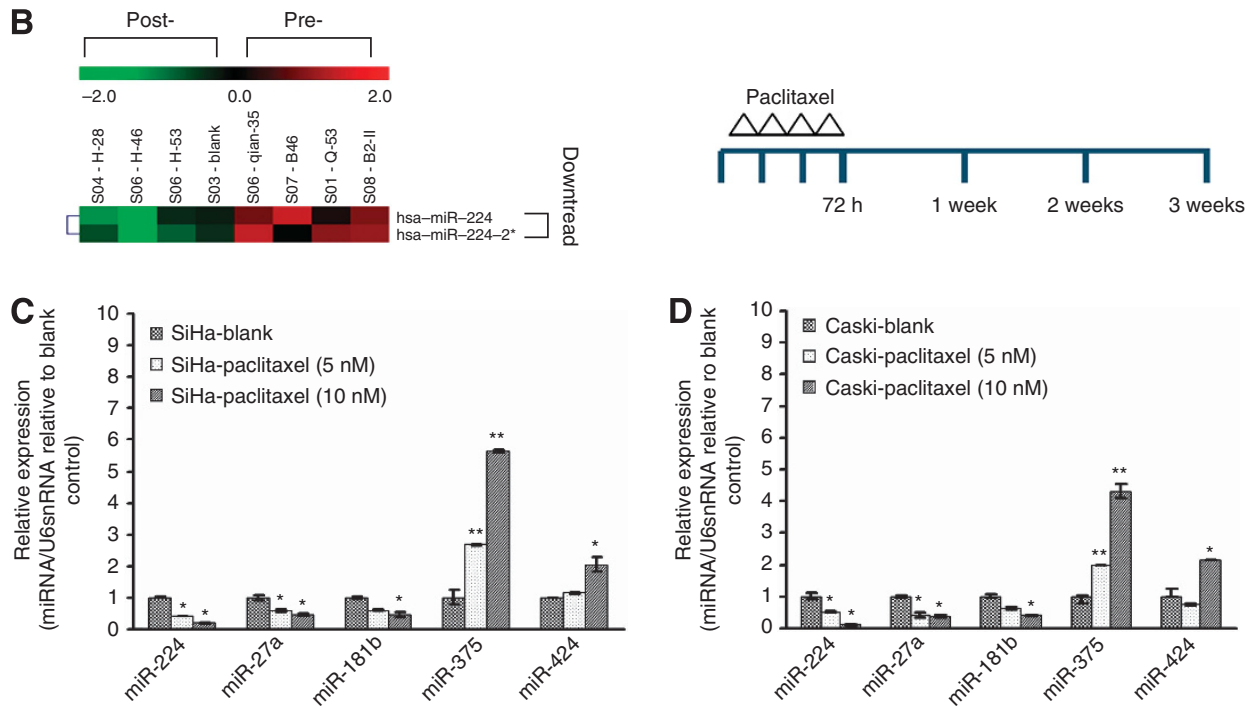

Figure 1. The association of differentially expressed miRNAs in cervical cancer tissues and cells with paclitaxel treatment. (A and B) Heat map showing the miRNA expression profiling and unsupervised hierarchical clustering analysis from four couples of self-paired cervical cancer tissues collected before and after chemotherapy (two cycles of intravenous neo-adjuvant chemotherapy, paclitaxel $135 \mathrm{mg} \mathrm{m}^{-2}$ and cisplatin $75 \mathrm{mg} \mathrm{m}^{-2}$, 3-week interval). Significantly differentially expressed miRNAs matched the threshold (differential $\geqslant 2$-fold, chip signal value $>500$ ) and statistical analysis standard were selected $(P<0.05$ in $\mathbf{A} ; P<0.01$ in $\mathbf{B})$. Each row represented the relative level of expression for a single miRNA and each column showed the expression level for a single sample. The red or green colour indicated relatively high or low expression, respectively. (C and D) Five differentially expressed miRNAs were validated in paclitaxel-treated cervical cancer cell lines SiHa (C) and Caski (D) using stem-loop real-time RT-PCR. There were two significantly upregulated (miR-375 and miR-424) and three significantly downregulated (miR-181b, miR-224, and miR-27a) miRNAs after paclitaxel treatment, consistent with the microarray results. (E) The expression of miR-375 in $\mathrm{SiHa}$ and Caski was measured by using RT-PCR at day 7, day 14, and day 21, respectively, after paclitaxel administration. MicroRNA expression values were rescaled relative to the blank control. All error bars indicated s.e.m. $\left({ }^{\star} P \leqslant 0.05,{ }^{\star} P \leqslant 0.01\right)$.

differentially expressed miRNAs existed between paclitaxel-sensitive and paclitaxel-resistant groups (Supplementary Figure S1).

To confirm the microarray findings, of above 21 differentially expressed miRNAs, 5 (miR-375, miR-424, miR-181b, miR-224, and miR-27a) were selected and validated in paclitaxel-treated cervical cancer cell lines (Caski and $\mathrm{SiHa}$ ). After cervical cancer cells were cultured with different amounts of paclitaxel $(0,5$, and $10 \mathrm{~nm}$ ) for $72 \mathrm{~h}$, the expression of miRNAs pre- and post-paclitaxel treatment was observed by using stem-loop real-time RT-PCR. The results showed that among the five differentially expressed miRNAs, miR-375 and miR-424 expressions were significantly upregulated as well as miR-181b, miR-224, and miR-27a expressions were significantly downregulated after paclitaxel treatment, which is consistent with the microarray results (Figure 1C and D). miR-375 was the most significantly upregulated with fold values range at $1.98-3.45$ and $4.3-5.89$ under 5 and $10 \mathrm{~nm}$ paclitaxel treatment, respectively, while miR-224 was the most significantly downregulated with fold values range at 1.91-2.05 and 6.3-7.89 under 5 and $10 \mathrm{~nm}$ paclitaxel treatment, respectively. These findings suggest that paclitaxel induces an increase in miR-375 expression in a clear dose-dependent manner.

To study further the impact of paclitaxel chemotherapy on the expression of miR-375 in cervical cancer cells, SiHa and Caski cells were cultured with $10 \mathrm{~nm}$ paclitaxel, after $72 \mathrm{~h}$, the paclitaxel was removed and the cells were cultured with complete medium continuously, subsequently the expression of miR-375 was measured by using RT-PCR at day 7 , day 14 , and day 21 , respectively. We found that paclitaxel induced progressive upregulated expression of miR-375 in SiHa and Caski cells, the upregulated expression of miR-375 reached a peak at day 7 after paclitaxel administration, and gradually declined until at about day 21 , and restored to the level before drug treatment. These findings suggest that the expression level of miR-375 in cervical cancer is paclitaxel dependent. 

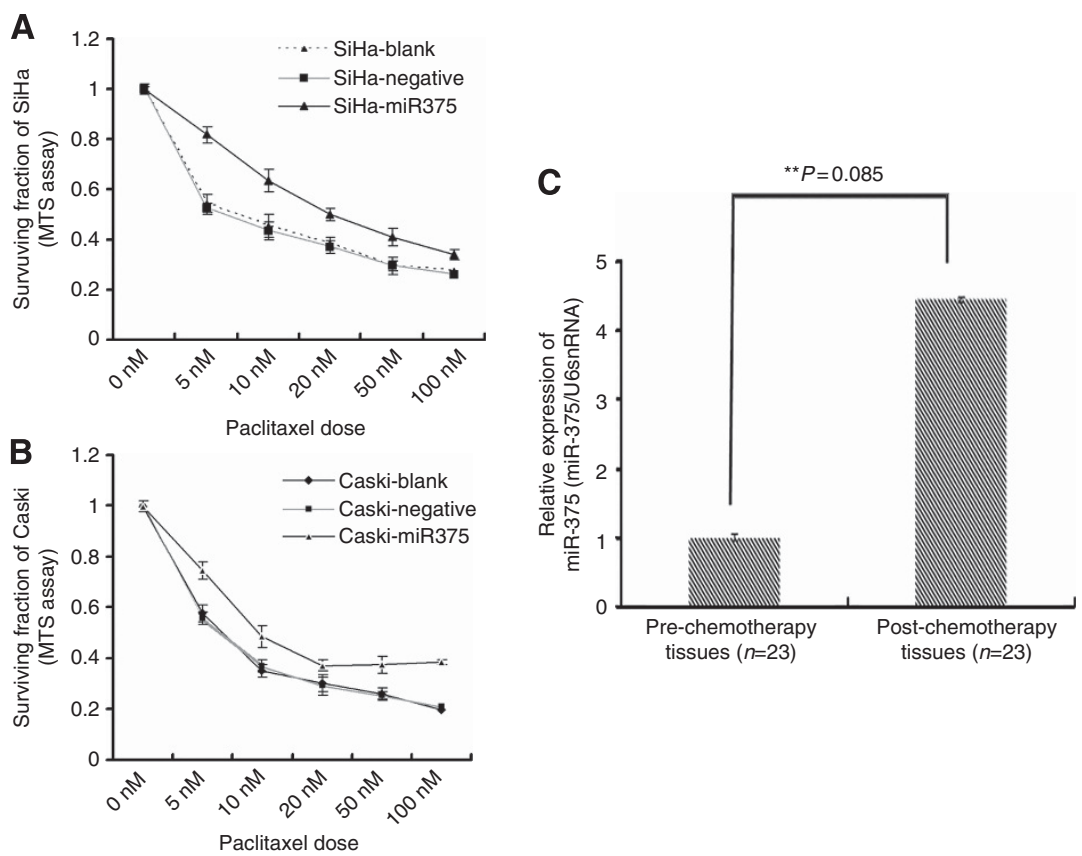

Figure 2. miR-375 expression is markedly increased in post-paclitaxel cervical cancer specimens and ectopically overexpressed miR-375 facilitates paclitaxel resistance in cervical cancer cells. (A and B) The expression of miR-375 was extraneously upregulated using a pre-miR-375 lentivirus transfection (MOI $=5$ for $\mathrm{SiHa} ; \mathrm{MOI}=10$ for Caski) and its impaction on paclitaxel sensitivity in cervical cancer cells (SiHa and Caski) at $72 \mathrm{~h}$ was observed using MTS. Paclitaxel sensitivities were significantly decreased after forced overexpression of miR-375 in SiHa (A) and Caski (B) cells compared with negative controls. Data were from at least triple biological and two technical replicates. (C) Real-time PCR validated the expression of miR-375 in 23 couples of self-paired pre- and post-chemotherapy cervical cancer tissues; miR-375 expression was markedly upregulated in the tissues after chemotherapy $(* \star P=0.0085)$.

miR-375 expression is markedly increase in post-paclitaxel cervical cancer specimens. To establish the clinical relevance of miR-375 regulation in paclitaxel chemotherapy, we therefore compared miR-375 expression in 23 couples of self-paired preand post-chemotherapy tissues from cervical squamous cell carcinoma patients who underwent neo-adjuvant chemotherapy before radical surgery and found that miR-375 was markedly unregulated (4.67-fold) in paclitaxel-treated cervical samples (Figure 2C). Our results imply that paclitaxel chemotherapy upregulates miR-375 expression in cervical cancer tissues and miR375 may be involved in the modulation of paclitaxel sensitivity in cervical cancer.

Exogenous miR-375 facilitates paclitaxel resistance in cervical cancer cells in vitro. To further confirm the involvement of miR375 in regulating paclitaxel sensitivity in cervical cancer cells, we exogenously upregulated miR-375 expression using a pre-miRNA lentiviral vector transfection and observed its impact on paclitaxel sensitivity using MTS. Cervical cancer cell lines (SiHa and Caski) in which miR-375 was expressed at a low level were transfected with pre-miR-375 or preNeg lentivirus vector and screened by flow cytometry after $96 \mathrm{~h}$. The stability of miR-375 expression in cells was investigated respectively by RT-PCR at 2 weeks, 3 weeks, and 1 month after transfection. The results showed a significant upregulation of miR-375 expression in $\mathrm{SiHa}$ and Caski cells transfected with pre-miR-375 lentivirus vector compared with the negative control, and the high miR-375 expression level continued for at least 1 month (Supplementary Figure S2). Furthermore, the drug sensitivity testing was determined with MTS assay at $72 \mathrm{~h}$ with different paclitaxel doses $(0,5,10,20,50$, and $100 \mathrm{~nm})$. As shown in Figure 2A and B, paclitaxel sensitivities were significantly decreased after forced overexpression of miR-375 in $\mathrm{SiHa}$ and Caski cells compared with miRNA-negative controls. The $\mathrm{IC}_{50}$ values were increased in $\mathrm{SiHa}$ and Caski with overexpression of miR-375 (45.81 \pm 1.33 and $20.18 \pm 0.72 \mathrm{~nm}$, respectively) compared with miRNA-negative controls $(8.54 \pm 1.52$ and $13.28 \pm 0.52 \mathrm{~nm}$, respectively) ( $\mathrm{SiHa}, P=0.023$; Caski, $P=0.035$ ). Our findings suggested that miR-375 negatively modulates the sensitivity to paclitaxel in cervical cancer cells.

The role of miR-375 in modulating paclitaxel resistance in vivo. After validated that miR-375 mediates paclitaxel resistance in different cervical cancer cell models, we injected subcutaneously the genetically modified (stably overexpressed miR-375) SiHa and Caski cells, as well as their negative and blank control cells, into 2- to 4-week female BALB/C nude mice, respectively. The rate of tumorigenesis reached $100 \%$. Once the mice developed palpable tumours (28 days for $\mathrm{SiHa}$ and 24 days for Caski) they were selected into the paclitaxel or saline treatment groups according to their closely matched tumour volumes. Then, paclitaxel $\left(15 \mathrm{mg} \mathrm{kg}^{-1}\right)$ and the same quantity of normal saline were intraperitoneally injected once a week for 4 weeks. As shown in Figure $3 \mathrm{~A}$ and $\mathrm{B}$, tumour growth was more significantly inhibited in negative control group than that in miR-375 overexpression group after paclitaxel administration. Meanwhile, tumour volume was shrinking at $78.4 \pm 3.21 \%$ in matched negative control mice after paclitaxel administration but only $25.3 \pm 1.42 \%$ in matched miR-375 overexpression group.

Seven days after complete paclitaxel treatment, tumours were removed, weighed, and sectioned for detecting miR-375 expression. In miR-375 overexpression group, excised tumour weight treated by saline was 1.15 -fold heavier than that by paclitaxel $(P=0.047)$, while in negative group, it achieved 2.35 -fold between saline and paclitaxel treatment $(P=0.003)$. The $\mathrm{H} \& \mathrm{E}$ staining of xenograft in $\mathrm{BALB} / \mathrm{c}$ mice showed the appearance of typical cervical carcinomas with tumour giant cells and allotypic nuclear 

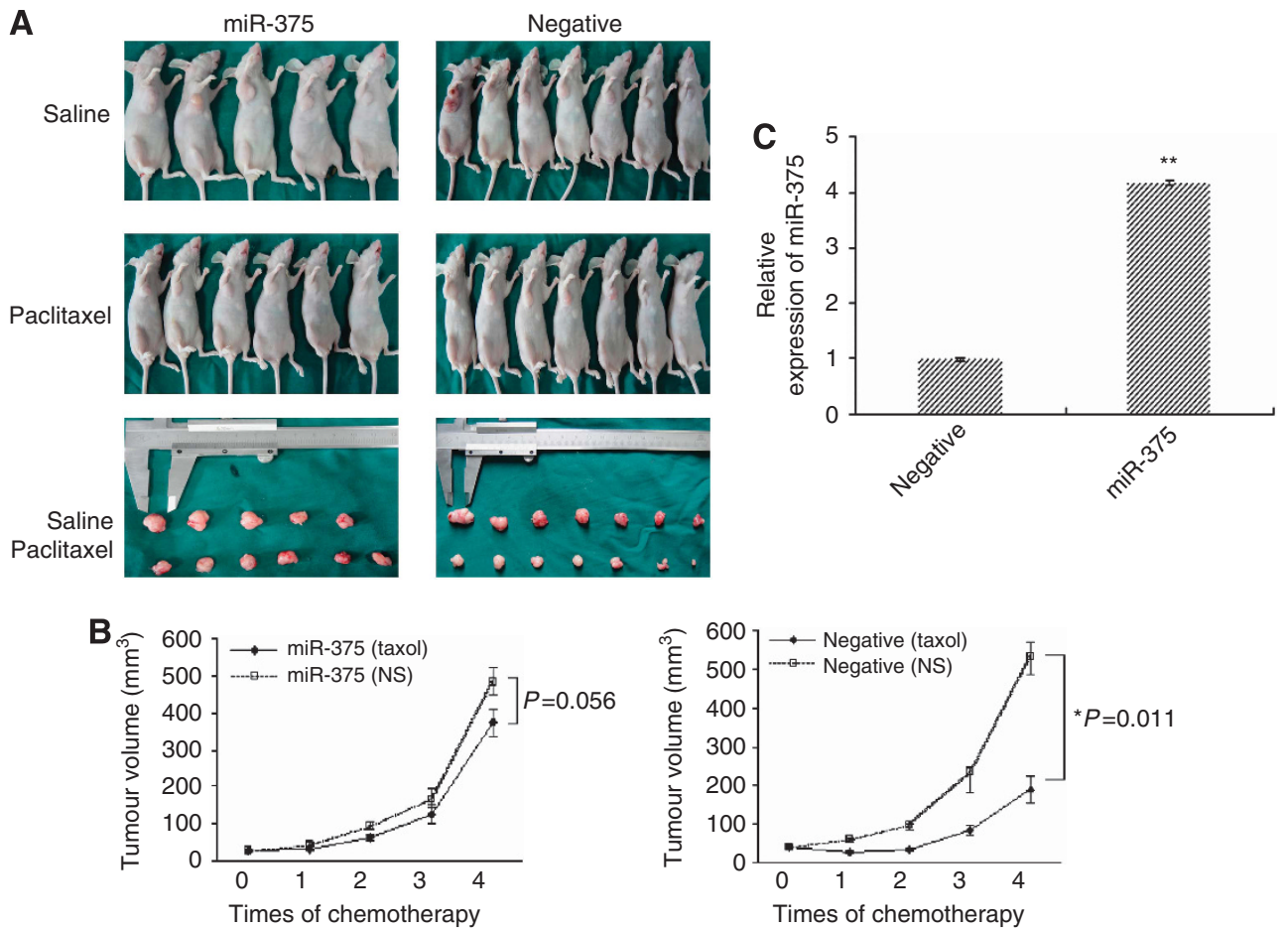

Figure 3. miR-375 mediates paclitaxel resistance in mouse models. BALB/c mice were subcutaneously inoculated with $4 \times 10^{6} \mathrm{SiHa}$ or $6 \times 10^{6}$ Caski cells with overexpressed miR-375 $(n=11)$, negative $(n=14)$, or blank controls $(n=7)$, respectively. After 28 days, transplanted tumours reached greater than $60-70 \mathrm{~mm}^{3}$, paclitaxel $\left(15 \mathrm{mg} \mathrm{kg}^{-1}\right)$ and the same quantity of normal saline were then intravenously injected into mice once a week for 4 weeks. Tumour volume $\left(\mathrm{mm}^{3}\right)$ was calculated as Width 2/ Length every 3 days. All mice were killed under anaesthesia on day 7 after complete drug treatments. Subcutaneous tumours were surgically excised, weighed, photographed, sectioned, and frozen for further investigation. (A and B) Impaired paclitaxel sensitivity in transplanted tumours in mice inoculated by miR-375-overexpressing SiHa cells was observed. (C) Real-time PCR analysis showed that miR-375 was upregulated in transplanted tumour tissues inoculated by miR-375-overexpressing SiHa cells. All error bars indicated s.e.m. ( $\left.{ }^{\star \star} P \leqslant 0.01,{ }^{\star} P \leqslant 0.05\right)$.

division, and tumour necrosis was observed in both paclitaxel groups. By using real-time RT-PCR, we observed that miR-375 expression was significantly increased in tumour tissues grafted by stably overexpressed miR-375 SiHa and Caski cells compared with those negative controls (Figure 3C). Thus, our findings in vivo further support that overexpressed miR-375 facilitates paclitaxel resistance in cervical cancer.

\section{DISCUSSION}

Although various mechanisms of chemo-resistance in cancers have been uncovered, the aberrant miRNA expression and its correlation with the chemo-resistance in cancers are still less understood. Accumulated evidence recently have shown that normal miRNA expression is closely linked to the sensitivity of cancer cells to anticancer drugs by targeting chemo-sensitivity-related genes $(\mathrm{Wu}$ and Xiao, 2009; Ren et al, 2010). Specific miRNA re-expression by miRNA mimics, or silence by synthetic antisense oligonucleotides, is able to alter chemotherapy sensitivity of cancer cells (Guo et al, 2010; Gong et al, 2011). Such studies imply that the exploration of the role of a specific miRNA and its mechanism in cancer chemoresistance may assist in discovering new approaches to reverse chemo-resistance of cancers. In the study, we profiled miRNA expression in cervical cancer tissues by miRNA microarray and compared their differential expression between paclitaxel sensitive and resistant and between self-paired pre- and post-paclitaxel chemotherapy. Totally, 21 differential miRNAs were found, including 13 downregulated and 8 upregulated between self-paired pre- and post-chemotherapy tissues. We did not found differential
miRNA expression between paclitaxel-resistant and -sensitive cancer tissues. The reasons partly may be due to inaccurately distinguish between sensitivity and resistance only after two cycles of paclitaxel chemotherapy in cervical cancer tissues, interpatient variability and heterogeneity, and too few paclitaxel-resistant tissues sent to microarray hybridisation, as well as the majority of tumours responds well initially to chemotherapy, $90 \%$ paclitaxel resistant were acquired drug resistance (Tanaka et al, 2012; Bamias et al, 2013).

Of 21 differential expressed miRNAs, 5 were selected to validate the findings in miRNA microarray in two cervical cancer cell lines treated with paclitaxel. We found that paclitaxel treatment remarkably altered miRNA expression of cervical cancer cells in a dose-dependent manner, of which miR-375 was the most significantly upregulated. Thus, the effect of miR-375 on chemoresistance in cervical cancer was further indentified. A role for miR-375 has initially been demonstrated in pancreatic development (Poy et al, 2009). Recently, miR-375 was identified as an important regulator in tumorigenesis and cancer progression; however, its exact functions remain largely inconsistent. The expression of miR-375 is downregulated in gastric cancer (Ding et al, 2010; Tsukamoto et al, 2010), head and neck squamous cell carcinoma (Hui et al, 2010), pharyngeal squamous cell carcinoma (Lajer et al, 2011), and prostate cancers (Szczyrba et al, 2011); additionally, entopic expression of miR-375 inhibited cancer cell proliferation, invasion, and cell motility (Mazar et al, 2011), suggesting that miR-375 could be a tumour suppressor. Conversely, the expression of miR-375 is upregulated in ER $\alpha$-positive breast cancer (de Souza Rocha Simonini et al, 2010), lung adenocarcinoma patients ( $\mathrm{Yu}$ et al, 2010), and HBV-positive HCC patients (Li et al, 2010), suggesting that it could be a tumour 
promoter. Thus, these conflicting reports regarding the association between miR-375 level and cancer suggested a more complex and possibly cancer-specific relationship. In our previous study, we reported that the expression of miR-375 was reduced in cervical cancer cells and overexpression of miR-375 significantly inhibited proliferation and blocked G1-S cell-cycle transition, which shows a tumour suppressor role of miR-375 in cervical cancer (Li et al, 2011; Wang et al, 2011). Here, we found an elevated miR-375 expression in cervical cancer tissues after paclitaxel chemotherapy by miRNA microarray. We further observed the association between miR-375 expression and paclitaxel chemotherapy in cervical cancer cell lines and tissues, and found that miR-375 expression was upregulated in cervical cancer cells following paclitaxel treatment in vitro and in tissues after paclitaxel combination chemotherapy. Thus, our findings suggest that paclitaxel may induce an acquired drug resistance in cervical cancer cells. It is possible that some of cervical cancer cells would survive under paclitaxel treatment, probably though upregulating miR-375 expression, then developing a potential to resist drug toxicity.

To verify our hypothesis, we further examined the effect of elevated miR-375 expression on paclitaxel resistance in cervical cancer cells, and found that enforced overexpression of miR-375 promoted paclitaxel resistance in cervical cancer cells in vitro. Moreover, we also observed, in mouse models with transplanted cervical cancer tumour by stable overexpression miR-375 cells, that transplanted tumour growth was slowed down and the tumour presented a remarkable resistance to paclitaxel administration. All of the findings are consistent with our previous report that miR375 was reduced in cervical cancer cells and overexpression of miR-375 significantly inhibited proliferation and blocked G1-S cell-cycle transition (Wang et al, 2011). Our results imply that chemotherapy usually cannot eliminates all the cancer cells and those residual cells that do not killed by drugs go into the status of tumour dormancy which is characterised by cell proliferation inhibition, cell-cycle arrest, as well as resistant to conventional chemotherapy (Lu et al, 2008; Yu and Zhu, 2013). Importantly, those dormant-like cells escaping from killing by drug may be reactivating with renewed miR-375 downregulation after drug withdrawal as we observed a de novo downregulation of miR-375 expression in cervical cancer cells after about 3 weeks of paclitaxel withdrawal in the study. Clinically, it is those revival cells that present the progression or recurrence and consequently contribute to poor prognosis of cancer patients. Our data suggest that chemotherapy kills most of actively proliferative cancer cells, but some of cells exposed to drug may develop a potential to decrease cellular proliferation for chemo-resistance by altering miRNA or other molecule expression.

As the function of miR-375 in tumorigenesis and cancer progression has cancer specificity, similar situation may exist in cancer chemo-resistance. Newly published data showed that miR375 expression was reduced in tamoxifen-resistant breast cancer cells compared with wild-type cells (Ward et al, 2013). But here, we validate the association between an elevated miR-375 expression induced by paclitaxel and the acquired paclitaxel resistance of cervical cancer in vivo and in vitro. This is the first report about the effect of miR-375 on chemo-resistance in cancer, to our knowledge. The conflicting results among different cancers and drugs might imply that miRNA function seems to be cancer specific and anticancer agent specific.

In summary, we find for the first time, to our knowledge, that paclitaxel may induce an acquired drug resistance in cervical cancer, that is, paclitaxel upregulates miR-375 expression and overexpressed miR-375 consequently produces chemo-resistance in cervical cancer in vitro and in vivo. miR-375 might be a therapeutic target in paclitaxel-resistant cervical cancer.

\section{ACKNOWLEDGEMENTS}

We thank Yuyan Mao and Caiyun Zhou for sample collections and technical assistance. We are grateful to Xiao Duan Chen for histological diagnosis of the cervical tissues. We should also like to acknowledge the continuous financial support from the National Natural Science Foundation of China (Grant Nos. 81172475 and 81172474), the Natural Science Foundation of Zhejiang Province of China (Grant Nos. Z2110056 and Y2110206).

\section{AUTHOR CONTRIBUTIONS}

YS conceived and carried out experiments. PW, YL, and FW carried out partial experiments and collected clinical samples. XW and WL conceived experiments and collected clinical samples. XC and FY analysed data. XX designed study and supervised experiments. All authors were involved in writing the paper and had final approval.

\section{REFERENCES}

Bamias A, Bamia C, Zagouri F, Kostouros E, Kakoyianni K, Rodolakis A, Vlahos G, Haidopoulos D, Thomakos N, Antsaklis A, Dimopoulos MA (2013) Improved survival trends in platinum-resistant patients with advanced ovarian, fallopian or peritoneal cancer treated with first-line paclitaxel/platinum chemotherapy: the impact of novel agents. Oncology 84: $158-165$.

Chang CJ, Chao CH, Xia W, Yang JY, Xiong Y, Li CW, Yu WH, Rehman SK, Hsu JL, Lee HH, Liu M, Chen CT, Yu D, Hung MC (2011) p53 regulates epithelial-mesenchymal transition and stem cell properties through modulating miRNAs. Nat Cell Biol 13: 317-323.

de Souza Rocha Simonini P, Breiling A, Gupta N, Malekpour M, Youns M, Omranipour R, Malekpour F, Volinia S, Croce CM, Najmabadi H, Diederichs S, Sahin O, Mayer D, Lyko F, Hoheisel JD, Riazalhosseini Y (2010) Epigenetically deregulated microRNA-375 is involved in a positive feedback loop with estrogen receptor alpha in breast cancer cells. Cancer Res 70: 9175-9184.

Bolstad BM, Irizarry RA, Astrandand M, Speed TP (2003) A comparison of normalization methods for high density oligonucleotide array data based on variance and bias. Bioinformatics19 185-193.

Dean M, Fojo T, Bates S (2005) Tumour stem cells and drug resistance. Nat Rev Cancer 5: 275-284.

Ding L, Xu Y, Zhang W, Deng Y, Si M, Du Y, Yao H, Liu X, Ke Y, Si J, Zhou T (2010) MiR-375 frequently downregulated in gastric cancer inhibits cell proliferation by targeting JAK2. Cell Res 20: 784-793.

Eisenhauer EA, Therasse P, Bogaerts J, Schwartz LH, Sargent D, Ford R, Dancey J, Arbuck S, Gwyther S, Mooney M, Rubinstein L, Shankar L, Dodd L, Kaplan R, Lacombe D, Verweij J (2009) New response evaluation criteria in solid tumours: revised RECIST guideline (version 1.1). Eur J Cancer 45: 228-247.

Gong C, Yao Y, Wang Y, Liu B, Wu W, Chen J, Su F, Yao H, Song E (2011) Up-regulation of miR-21 mediates resistance to trastuzumab therapy for breast cancer. J Biol Chem 286: 19127-19137.

Guo L, Liu Y, Bai Y, Sun Y, Xiao F, Guo Y (2010) Gene expression profiling of drug-resistant small cell lung cancer cells by combining microRNA and cDNA expression analysis. Eur J Cancer 46: 1692-1702.

Hui AB, Lenarduzzi M, Krushel T, Waldron L, Pintilie M, Shi W, PerezOrdonez B, Jurisica I, O'Sullivan B, Waldron J, Gullane P, Cummings B, Liu FF (2010) Comprehensive MicroRNA profiling for head and neck squamous cell carcinomas. Clin Cancer Res 16: 1129-1139.

Kim CH, Kim HK, Rettig RL, Kim J, Lee ET, Aprelikova O, Choi IJ, Munroe DJ, Green JE (2011) miRNA signature associated with outcome of gastric cancer patients following chemotherapy. BMC Med Genomics 4: 79.

Lajer CB, Nielsen FC, Friis-Hansen L, Norrild B, Borup R (2011) Different miRNA signatures of oral and pharyngeal squamous cell carcinomas: a prospective translational study. Br J Cancer 104: 830-840. 
Li LM, Hu ZB, Zhou ZX, Chen X, Liu FY (2010) Serum microRNA profiles serve as novel biomarkers for HBV infection and diagnosis of HBV positive hepatocarcinoma. Cancer Res 70: 9798-9807.

Li Y, Wang F, Xu J, Ye F, Shen Y, Zhou J, Lu W, Wan X, Ma D, Xie X (2011) Progressive miRNA expression profiles in cervical carcinogenesis and identification of HPV-related target genes for miR-29. J Pathol 224: 484-495.

Lu Z, Luo RZ, Lu Y, Zhang X, Yu Q, Khare S, Kondo S, Kondo Y, Yu Y, Mills GB, Liao WS, Bast Jr. RC (2008) The tumor suppressor gene ARHI regulates autophagy and tumor dormancy in human ovarian cancer cells. $J$ Clin Invest 118: 3917-3929.

Mazar J, DeBlasio D, Govindarajan SS, Zhang S, Perera RJ (2011) Epigenetic regulation of microRNA-375 and its role in melanoma development in humans. FEBS Lett 585: 2467-2476.

Meng F, Henson R, Lang M, Wehbe H, Maheshwari S, Mendell JT, Jiang J, Schmittgen TD, Patel T (2006) Involvement of human micro-RNA in growth and response to chemotherapy in human cholangiocarcinoma cell lines. Gastroenterology 130: 2113-2129.

Nakajima G, Hayashi K, Xi Y, Kudo K, Uchida K, Takasaki K, Yamamoto M, Ju J (2006) Non-coding MicroRNAs hsa-let-7g and hsa-miR-181b are associated with chemoresponse to S-1 in colon cancer. Cancer Genomics Proteomics 3: 317-324.

Poy MN, Hausser J, Trajkovski M, Braun M, Collins S, Rorsman P, Zavolan M, Stoffel M (2009) miR-375 maintains normal pancreatic alpha- and beta-cell mass. Proc Natl Acad Sci USA 106: 5813-5818.

Ranganathan AC, Zhang L, Adam AP, Aguirre-Ghiso JA (2006) Functional coupling of $\mathrm{p} 38$-induced up-regulation of $\mathrm{BiP}$ and activation of RNAdependent protein kinase-like endoplasmic reticulum kinase to drug resistance of dormant carcinoma cells. Cancer Res 66: 1702-1711.

Ren Y, Zhou X, Mei M, Yuan XB, Han L, Wang GX, Jia ZF, Xu P, Pu PY, Kang CS (2010) MicroRNA-21 inhibitor sensitizes human glioblastoma cells U251 (PTEN-mutant) and LN229 (PTEN-wild type) to taxol. BMC Cancer 10: 27.

Szczyrba J, Nolte E, Wach S, Kremmer E, Stohr R, Hartmann A, Wieland W, Wullich B, Grasser FA (2011) Downregulation of Sec23A protein by miRNA-375 in prostate carcinoma. Mol Cancer Res 9: 791-800.

Tanaka T, Toujima S, Tanaka J (2012) Differential sensitivity to paclitaxelinduced apoptosis and growth suppression in paclitaxel-resistant cell lines established from HEC-1 human endometrial adenocarcinoma cells. Int $J$ Oncol 41: 1837-1844.

Tsukamoto Y, Nakada C, Noguchi T, Tanigawa M, Nguyen LT, Uchida T, Hijiya N, Matsuura K, Fujioka T, Seto M, Moriyama M (2010) MicroRNA-375 is downregulated in gastric carcinomas and regulates cell survival by targeting PDK1 and 14-3-3zeta. Cancer Res 70: 2339-2349.

Wang F, Li Y, Zhou J, Xu J, Peng C, Ye F, Shen Y, Lu W, Wan X, Xie X (2011) miR-375 is down-regulated in squamous cervical cancer and inhibits cell migration and invasion via targeting transcription factor SP1. Am J Pathol 179: $2580-2588$.

Wang JW, Cheng JQ (2008) A simple method for profiling miRNA expression. Methods Mol Biol 414: 183-190.

Wang Z, Li Y, Ahmad A, Azmi AS, Kong D, Banerjee S, Sarkar FH (2010) Targeting miRNAs involved in cancer stem cell and EMT regulation: An emerging concept in overcoming drug resistance. Drug Resist Updat 13: $109-118$.

Ward A, Balwierz A, Zhang JD, Küblbeck M, Pawitan Y, Hielscher T, Wiemann S, Sahin O (2013) Re-expression of microRNA-375 reverses both tamoxifen resistance and accompanying EMT-like properties in breast cancer. Oncogene 32: 1173-1182.

Wendt MK, Smith JA, Schiemann WP (2010) Transforming growth factorbeta-induced epithelial-mesenchymal transition facilitates epidermal growth factor-dependent breast cancer progression. Oncogene 29: 6485-6498.

Wilson CM, Tobin S, Young RC (2004) The exploding worldwide cancer burden: the impact of cancer on women. Int J Gynecol Cancer 14 1-11.

Workman P, Aboagye EO, Balkwill F, Balmain A, Bruder G, Chaplin DJ, Double JA, Everitt J, Farningham D, Glennie MJ, Kelland LR, Robinson V, Stratford IJ, Tozer GM, Watson S, Wedge SR, Eccles SA (2010) An ad hoc committee of the National Cancer Research Institute Guidelines for the welfare and use of animals in cancer research. Br J Cancer 102: 1555-1577.

Wu X, Xiao H (2009) miRNAs modulate the drug response of tumor cells. Sci China C Life Sci 52: 797-801.

Yavas G, Yavas C (2012) Comment on 'Quality of life and emotional distress in early stage and locally advanced cervical cancer patients: a prospective, longitudinal study' by Ferrandina et al. (GYNECOL ONCOL 2012; 124:389-394). Gynecol Oncol 126: 167 author reply 167-168.

Yu Y, Zhu Z (2013) Cell dormancy and tumor refractory. Anticancer Agents Med Chem 13: 199-202.

Yu L, Todd NW, Xing L, Xie Y, Zhang H (2010) Early detection of lung adenocarcinoma in sputum by a panel of microRNA markers. Int $J$ Cancer 127: $2870-2878$.

Zou Z, Wu L, Ding H, Wang Y, Zhang Y, Chen X, Zhang CY, Zhang Q, Zen K (2012) MicroRNA-30a sensitizes tumor cells to cis-platinum via suppressing beclin 1-mediated autophagy. J Biol Chem 287: 4148-4156.

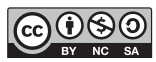

This work is licensed under the Creative Commons Attribution-NonCommercial-Share Alike 3.0 Unported License. To view a copy of this license, visit http://creativecommons. org/licenses/by-nc-sa/3.0/

Supplementary Information accompanies this paper on British Journal of Cancer website (http://www.nature.com/bjc) 